\section{Smarter microscopes}

Innovations in acquisition and analysis are transforming microscopy.

Despite substantial advances in microscope optics and computational resources, many microscopy experiments are still carried out much as they were decades ago, with samples being prepared and imaged one by one, zeroed in on by a trained user, and recorded in just enough numbers to be considered scientifically rigorous. Although this approach is undoubtedly powerful, it requires abundant hands-on time and expertise, can be limited in terms of statistics, and can be biased by the imaging of structures that match an expectation.

There has been a shift away from fully manual imaging that is poised to eventually take humans out of the loop in imaging experiments. For example, technological developments in robotics for handling biological samples have made many types of experiments high throughput. Combined with software and hardware tools developed to automate high-throughput imaging, it is easy to imagine a world where human hands aren't required for sample preparation, loading and image acquisition. Super-resolution singlemolecule localization microscopy is one area where such automated strategies are beginning

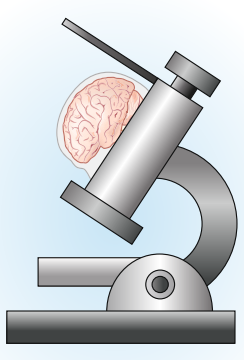

The 'smart' microscope will improve imaging. Credit: Marina Corral Spence/Springer Nature

to bear fruit (Opt. Express 26, 30882-30900, 2018; Nat. Commun. 10, 1223, 2019; Nat. Methods 14, 1184-1190, 2017).

Automated acquisition has benefits beyond ease and throughout, and can lead to higher quality images. A core principle of 'smart microscopy' is that the microscope and acquisition controls interact with each other to create positive feedback. One notable example is the Autopilot framework for adaptive imaging, which has enabled time-lapse imaging of the development of zebrafish, fruit fly and mouse embryos
(Nat. Biotechnol. 34, 1267-1278, 2016; Cell 175, 859-876.e33, 2018). In this case, the microscope control software actively manages aspects such as the position and $3 \mathrm{D}$ orientation of the sample and acquisition parameters in real time to optimize speed, quality and consistency of imaging over time.

Beyond acquiring images, several user-friendly tools are established for semi- and fully automated analysis for most standard tasks, including segmentation and phenotyping, and even more detailed quantitative analyses. These methods are under constant development and enable very sophisticated analyses of complex samples.

We think smart microscopy will be an important trend in years to come; innovation will be spurred by improvements to and seamless integration of all the aspects listed above. Deep machine learning is likely to be pivotal in such improvements, as well as in strategies for handling the data deluge associated with such imaging. We also hope that at the heart of such advances will be benefits to the health of samples, as rightly stressed in a Commentary on this topic (Nat. Biotechnol. 33, 815-818, 2015).

\section{Rita Strack}

Published online: 6 January 2020 https://oi.org/10.1038/s41592-019-0708-0

\section{Informatics for genomics}

Advanced algorithms evolve in the age of genomic big data.

Genetic variation underlies many complex phenotypes and diseases in human populations. The fast-moving development of high-throughput sequencing technologies opens up opportunities to identify genetic variation on an ever-more-comprehensive scale, in unprecedentedly large cohorts. This raises unceasing demands for advanced informatics methods for genomic data analysis.

One challenge is the handling of huge sequencing datasets (Nat. Rev. Genet. 20, 693-701, 2019). The reference genome has been pivotal in identifying and analyzing genetic variants since the completion of the Human Genome Project. However, it has become ever clearer that a referencegenome-centric strategy may be neither sufficient to characterize the full spectrum of human genomic variation nor efficient for computational and statistical analysis. A variety of graph-based models have

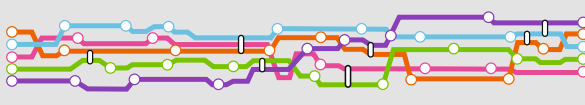

Some genome graphs resemble subway maps. Credit: Marina Corral Spence/Springer Nature

emerged recently that achieve higher accuracy and speed (Nat. Genet. 49, 16541660, 2017; Nat. Biotechnol. 36, 875-879, 2018; Nat. Genet. 51, 354-362, 2019). Other architectures take advantage of genealogical relationship between samples in human populations (Nat. Genet. 51, 1330-1338, 2019). We expect that the coming years will see more powerful models, as well as integration and update with the vast arsenal of genomic analysis tools.

Another direction of method development is driven by the imperfection of sequencing technologies. Despite continuous improvement, the trade-off between factors such as coverage, read length and error rate still limits the quantity and quality of the data that are available to genome informatics tools. Multi-platform strategies have shown promising performance in tasks such as de novo genome assembly and structural variation identification (Nat. Commun. 10, 1784, 2019). We look forward to informatics approaches keeping pace with the advancement of sequencing technologies (Nat. Biotechnol. 37, 1155-1162, 2019).

On top of the above two areas, the accurate and comprehensive interpretation of human genomic data in terms of biological and medical significance is arguably the holy grail of human genomics. Statistical and machine-learning methods have been thriving in the areas of statistical genetics and functional genomics. With the deluge of large-scale genomic data, variant types previously understudied (due to their low frequency or complex structure) are now becoming the next frontier of genetic investigation. We believe informatics tools are indispensable for exploring the sea of genomic big data.

Lin Tang

Published online: 6 January 2020 https://doi.org/10.1038/s41592-019-0709-z 